\title{
781.
}

\section{ON THE AUTOMORPHIC TRANSFORMATION OF THE BINARY CUBIC FUNCTION.}

[From the Proceedings of the London Mathematical Society, vol. xIv. (1883), pp. 103-108. Read Jan. 11, 1883.]

I CONSIDER the cubic equation $\left(a, b, c, d \gamma(x, 1)^{3}=0\right.$. It is shown (Serret, Cours d'Algèbre supérieure, 4th ed., Paris, 1879, t. II. pp. 466-471) how, given one root of the equation, the other two roots can be each of them expressed rationally in terms of this root and of the square root of the discriminant; viz. making the proper changes of notation, and writing

$$
\begin{gathered}
A, B, C=a c-b^{2}, a d-b c, b d-c^{2}, \lambda=\sqrt{-\frac{1}{3}} \\
\Omega=B^{2}-4 A C,=a^{2} d^{2}+4 a c^{3}+4 b^{3} d-3 b^{2} c^{2}-6 a b c d, \\
\alpha=\frac{-\lambda \sqrt{ } \Omega+B}{2 \lambda \sqrt{ } \Omega}, \beta=\frac{+2 C}{2 \lambda \sqrt{ } \Omega}, \gamma=\frac{-2 A}{2 \lambda \sqrt{ } \Omega}, \delta=\frac{-\lambda \sqrt{ } \Omega-B}{2 \lambda \sqrt{ } \Omega},
\end{gathered}
$$

(values which give $\alpha+\delta=-1, \alpha \delta-\beta \gamma=-1$, and therefore also

$$
\alpha^{2}+\alpha \delta+\delta^{2}+\beta \gamma=0,
$$

which is the condition in order that the function $\phi x,=\frac{\alpha x+\beta}{\gamma x+\delta}$, may be periodic of the third order, $\left.\phi^{3} x=x\right)$, then, $u$ being a root of the equation, say $\left(a, b, c, d \gamma(u, 1)^{3}=0\right.$, the other two roots are

and

$$
\phi u,=\frac{\alpha u+\beta}{\gamma u+\delta},
$$

$$
\phi^{2} u,=\phi^{-1} u,=\frac{\left(\alpha^{2}+\beta \gamma\right) u+\beta(\alpha+\delta)}{\gamma(\alpha+\delta) u+\delta^{2}+\beta \gamma},=\frac{\delta u-\beta}{-\gamma u+\alpha},
$$

where observe that, by the change of $\sqrt{ } \Omega$ into $-\sqrt{ } \Omega, \alpha, \beta, \gamma, \delta$ become $\delta,-\beta,-\gamma, \alpha$; so that the last-mentioned value $\phi^{-1} u$ is, in fact, the value obtained from $\phi u$ by the mere change of sign of the radical. 
It is to be observed that, if we have between two roots $u, v$ of the equation

$$
\left(a, b, c, d \gamma(x, 1)^{3}=0,\right.
$$

a relation $v=\frac{\alpha u+\beta}{\gamma u+\delta}$, where $\alpha, \beta, \gamma, \delta$ have given values, this implies in the first place a relation between $a, b, c, d$ (and the given values of $\alpha, \beta, \gamma, \delta$ ), and it implies moreover that $u$, and consequently also $v$, are not any roots whatever, but two determinate roots of the equation; viz. $u, v$ will be each of them expressible rationally in terms of $a, b, c, d$ and $\alpha, \beta, \gamma, \delta$. And if, in order that $(a, b, c, d)$ may remain arbitrary, we consider $\alpha, \beta, \gamma, \delta$ as given quantities satisfying the relation which exists between these quantities and $(a, b, c, d)$, then in general we still have $u, v$ determinate roots of the cubic equation. But in the foregoing solution $u$ is any root whatever of the cubic equation.

To examine how this is, starting from the equations

we have

$$
(a, b, c, d \gamma u, 1)^{3}=0, \quad\left(a, b, c, d \gamma(v, 1)^{3}=0, \quad v=\frac{\alpha u+\beta}{\gamma u+\delta},\right.
$$

and therefore

$$
a\left(u^{3}-v^{3}\right)+3 b\left(u^{2}-v^{2}\right)+3 c(u-v)=0,
$$

that is,

$$
a\left(u^{2}+u v+v^{2}\right)+3 b(u+v)+3 c=0,
$$

$$
a v^{2}+(a u+3 b) v+a u^{2}+3 b u+3 c=0 ;
$$

or, writing herein for $v$ its value,

that is,

$$
a(\alpha u+\beta)^{2}+(\alpha u+\beta)(\gamma u+\delta)(a u+3 b)+(\gamma u+\delta)^{2}\left(a u^{2}+3 b u+3\right)=0 ;
$$

$$
\begin{aligned}
a(\alpha u+\beta)^{2} & \quad \alpha \gamma\left(a u^{3}+3 b u^{2}\right)+\gamma^{2}\left(a u^{4}+3 b u^{3}+3 c u^{2}\right) \\
& +(\alpha \delta+\beta \gamma)\left(a u^{2}+3 b u\right)+2 \gamma \delta\left(a u^{3}+3 b u^{2}+3 c u\right) \\
& +\quad \beta \delta(a u+3 b)+\delta^{2}\left(a u^{2}+3 b u+c\right)=0
\end{aligned}
$$

or, reducing by the equation $a u^{3}+3 b u^{2}+3 c u+d=0$, this is

$$
\begin{aligned}
a(\alpha u+\beta)^{2} & +\quad \alpha \gamma(-3 c u-d)+\gamma^{2}(-d u) \\
& +(\alpha \delta+\beta \gamma)\left(a u^{2}+3 b u\right)+2 \gamma \delta(-d) \\
& +\quad \beta \delta(a u+3 b)+\delta^{2}\left(a u^{2}+3 b u+3 c\right)=0
\end{aligned}
$$

and, collecting the terms, this is

$$
\begin{aligned}
& u^{2} a\left(\alpha^{2}+\alpha \delta+\delta^{2}+\beta \gamma\right) \\
+ & u\left\{a(2 \alpha \beta+\beta \delta)+3 b\left(\alpha \delta+\delta^{2}+\beta \gamma\right)-3 c \alpha \gamma-d \gamma^{2}\right\} \\
+ & a \beta^{2}+3 b \beta \delta+3 c \delta^{2}+d(-\alpha \gamma-2 \gamma \delta)=0 .
\end{aligned}
$$

We can, from this equation, and the equation $a u^{3}+3 b u^{2}+3 c u+d=0$, eliminate $u$, thus obtaining a relation between $a, b, c, d, \alpha, \beta, \gamma, \delta$; and, this relation being satisfied, the two equations then determine $u$ rationally in terms of these quantities. 
We may without loss of generality assume $\alpha \delta-\beta \gamma=1$; and, this being so, if we then further assume $\alpha+\delta=-1$, then we have

$$
\alpha^{2}+\alpha \delta+\delta^{2}+\beta \gamma=0
$$

which is, as above appearing, the condition for $\phi^{3} x=0$. The foregoing equation in $u$ thus becomes

$$
\begin{aligned}
& u\left\{a \beta(\alpha-1)-3 b \alpha^{2}-3 c \alpha \gamma-d \gamma^{2}\right\} \\
& +\left\{a \beta^{2}+3 b \beta \delta+3 c \delta^{2}-d \gamma(\delta-1)\right\}=0 ;
\end{aligned}
$$

a linear equation giving (in a simplified form) the like results to those given by the quadric equation; viz. substituting in the cubic equation the value of $u$ given by the linear equation, we have a relation between $a, b, c, d, \alpha, \beta, \gamma, \delta$; and, this relation being satisfied, $u$ has the determinate value given by the linear equation.

The only way in which $u$ can cease to have this determinate value, and so be capable of being any root whatever of the cubic equation, is when the linear equation becomes $0=0$; viz. if

$$
\begin{aligned}
& a \beta(\alpha-1)-3 b \alpha^{2}-3 c \alpha \gamma-d \gamma^{2}=0, \\
& a \beta^{2}+3 b \beta \delta+3 c \delta^{2}-d \gamma(\delta-1)=0,
\end{aligned}
$$

equations which are, in fact, satisfied by the foregoing values of $\alpha, \beta, \gamma, \delta$, as may be verified without difficulty.

It is to be remarked that if, instead of the root $u$ and the equation $v=\frac{\alpha u+\beta}{\gamma u+\delta}$, we consider the root $v$ and the equation $u=\frac{\delta v-\beta}{-\gamma v+\alpha}$; then, instead of $\alpha, \beta, \gamma, \delta$, we have $\delta,-\beta,-\gamma, \alpha$, and the corresponding equations are

$$
\begin{aligned}
& a \beta(\delta-1)+3 b \delta^{2}+3 c \gamma \delta+d \gamma^{2}=0, \\
& a \beta^{2}+3 b \alpha \beta+3 c \alpha^{2}+d \gamma(\alpha-1)=0,
\end{aligned}
$$

equations which are also satisfied by the foregoing values of $\alpha, \beta, \gamma, \delta$. And the four equations, together with $\alpha \delta-\beta \gamma=1$ and $\alpha+\delta=1$, are more than sufficient to determine the foregoing values of $\alpha, \beta, \gamma, \delta$.

But we further verify without difficulty that the foregoing values of $\alpha, \beta, \gamma, \delta$ give identically

$$
(a, b, c, d \gamma \alpha \alpha x+\beta y, \gamma x+\delta y)^{3}=\left(a, b, c, d \gamma(x, y)^{3} ;\right.
$$

or the formulæ lead to an automorphic transformation of the binary cubic $\left(a, b, c, d \gamma(x, y)^{3}\right.$. And conversely, starting from the notion of the automorphic transformation of the binary cubic, we ought to be able to obtain the foregoing formulæ.

For greater convenience, I write the equation of transformation in the form

$$
(a, b, c, d \gamma \alpha x+\beta y, \gamma x+\delta y)^{3}=-\theta\left(a, b, c, d \gamma(x, y)^{3} ;\right.
$$


the equations to be satisfied by $\alpha, \beta, \gamma, \delta, \theta$ then are

$$
\begin{aligned}
& a \alpha^{3}+b .3 \alpha^{2} \gamma+c .3 \alpha \gamma^{2}+d \gamma^{3}=-a \theta, \\
& a \alpha^{2} \beta+b\left(\alpha^{2} \delta+2 \alpha \beta \gamma\right)+c\left(2 \alpha \gamma \delta+\beta \gamma^{2}\right)+d \gamma^{2} \delta=-b \theta, \\
& a \alpha \beta^{2}+b\left(2 \alpha \beta \delta+\beta^{2} \gamma\right)+c\left(\alpha \delta^{2}+2 \beta \gamma \delta\right)+d \delta^{2}=-c \theta, \\
& a \beta^{3}+b .3 \beta^{2} \delta+c .3 \beta \delta^{2}+d \delta^{3}=-d \theta .
\end{aligned}
$$

Writing $\alpha \delta-\beta \gamma=\nabla$, and as before $\Omega$ for the discriminant, the theory of invariants gives $\Omega \nabla^{6}=\Omega \theta^{4}$. We are considering the case of the general cubic function $(a, b, c, d \gamma x, y)^{3}$, for which $\Omega$ is not $=0$; and we have therefore $\nabla^{6}-\theta^{4}=0$, or, what is the same thing, we may write $\nabla=q^{2}, \theta=q^{3}$, where $q$ is arbitrary.

It is to be shown that $\alpha+\delta$ is $=q$ or $-2 q$, the latter, value giving the trivial solution $\alpha x+\beta y, \gamma x+\delta y=(x, y)$. The proper solution thus corresponds to $\nabla=q^{2}, \alpha+\delta=q$, that is,

$$
(\alpha+\delta)^{2}-(\alpha \delta-\beta \gamma)=0, \text { or } \alpha^{2}+\delta^{2}+\alpha \delta+\beta \gamma=0,
$$

the condition for the periodic function $\phi^{3} x-x=0$.

For this purpose, from the foregoing equations eliminating $a, b, c, d$, we have

$$
\begin{array}{cccc}
\alpha^{3}+\theta, & 3 \alpha^{2} \gamma, & 3 \alpha \gamma^{2}, & \gamma^{3} \\
\alpha^{2} \beta, & \alpha^{2} \delta+2 \alpha \beta \gamma+\theta, & 2 \alpha \gamma \delta+\beta \gamma^{2}, & \gamma^{2} \delta \\
\alpha \beta^{2}, & 2 \alpha \beta \delta+\beta^{2} \gamma, & \alpha \delta^{2}+2 \beta \gamma \delta+\theta, & \gamma \delta^{2} \\
\beta^{3}, & 3 \beta^{2} \delta, & 3 \beta \delta^{2}, & \delta^{3}+\theta
\end{array} \mid=0 ;
$$

an equation which may be written

$$
+\theta(123+234+341+412)+\theta^{2}(12+23+34+41+13+42)+\theta^{3}(1+2+3+4)+\theta^{4}=0,
$$

where 123, \&c., are the first diagonal minors, 12, \&c., the second diagonal minors, 1, \&c., the third diagonal minors, or diagonal terms of the foregoing determinant, writing therein $\theta=0$. We find without difficulty

$$
1,2,3,4=\alpha^{3}, \alpha^{2} \delta+2 \alpha \beta \gamma, \alpha \delta^{2}+2 \beta \gamma \delta, \delta^{3},
$$

$12,13,14,23,24,34=\left\{\alpha^{4}, \alpha^{2}(\alpha \delta+3 \beta \gamma),\left(\alpha^{2} \delta^{2}+\alpha \delta \beta \gamma+\beta^{2} \gamma^{2}\right)\right.$,

$$
\left.\left(\alpha^{2} \delta^{2}+\alpha \delta \beta \gamma+\beta^{2} \gamma^{2}\right), \delta^{2}(\alpha \delta+3 \beta \gamma), \delta^{4}\right\} \nabla,
$$

$123,124,134,234=\left\{\alpha^{3}, \alpha(\alpha \delta+2 \beta \gamma), \delta(\alpha \delta+2 \beta \gamma), \delta^{3}\right\} \nabla^{3}$,

$$
=\nabla^{6} \text {, }
$$

and the equation thus is

$$
\begin{aligned}
& \theta^{4} \\
+ & \theta^{3}\left[\alpha^{3}+\alpha^{2} \delta+\alpha \delta^{2}+\delta^{3}+2 \alpha \beta \gamma+2 \beta \gamma \delta\right] \\
+ & \theta^{2} \nabla\left[\alpha^{4}+\alpha^{3} \delta+\alpha \delta^{3}+\delta^{4}+3 \alpha^{2} \beta \gamma+3 \beta \gamma \delta^{2}+2 \alpha^{2} \delta^{2}+2 \alpha \delta \beta \gamma+2 \beta^{2} \gamma^{2}\right] \\
+ & \theta \nabla^{3}\left[\alpha^{3}+\delta^{3}+(\alpha+\delta)(\alpha \delta+2 \beta \gamma)\right] \\
+ & \nabla^{6}=0 .
\end{aligned}
$$


Putting herein $\alpha+\delta=m, \alpha \delta=n, \beta \gamma=n-\nabla$, it is found that $n$ disappears altogether from the equation; viz. the resulting form is

$$
\theta^{4}+\theta^{3} m\left(m^{2}-2 \nabla\right)+\theta^{2} \nabla\left(m^{4}-3 \nabla m^{2}+2 \nabla^{2}\right)+\theta \nabla^{3} m\left(m^{2}-2 \nabla\right)+\nabla^{6}=0,
$$

or, what is the same thing,

$$
m^{4} \cdot \theta^{2} \nabla+m^{3}\left(\theta^{3}+\theta \nabla^{3}\right)-3 \theta^{2} \nabla^{2} m^{2}-2\left(\theta \nabla^{4}+\theta^{3} \nabla\right) m+\theta^{4}+2 \theta^{2} \nabla^{3}+\nabla^{6}=0 .
$$

Putting for $\theta, \nabla$, their values $q^{3}, q^{2}$, the equation divides by $q^{8}$, and omitting this factor it becomes

viz. this is

$$
m^{4}+2 m^{3} q-3 m^{2} q^{2}-4 m q^{3}+4 q^{4}=0 ;
$$

or we have

$$
\{(m-q)(m+2 q)\}^{2}=0,
$$

$$
m=q \text { or }-2 q \text {; that is, } \alpha+\delta=q \text {, or } \alpha+\delta=-2 q \text {. }
$$

Writing; as before, $A, B, C=a c-b^{2}, a d-b c, b d-c^{2}$, we deduce from the foregoing equations

$$
\begin{aligned}
& \theta^{2} A=\nabla^{2}\left[A \alpha^{2}+B \cdot \alpha \gamma+C \gamma^{2}\right], \\
& \theta^{2} B=\nabla^{2}[A \cdot 2 \alpha \beta+B(\alpha \delta+\beta \gamma)+C \cdot 2 \gamma \delta], \\
& \theta^{2} C=\nabla^{2}\left[A \beta^{2}+B . \beta \delta+C \delta^{2}\right],
\end{aligned}
$$

which are, in fact, the equations for the automorphic transformation of the Hessian $\left(A, B, C \gamma(x, y)^{2}\right.$. And, writing herein $\theta, \nabla=q^{3}, q^{2}$, the equations become

$$
\begin{array}{ll}
A\left(\alpha^{2}-q^{2}\right)+B \alpha \gamma+C \cdot \gamma^{2} & =0, \\
A 2 \alpha \beta+B\left(\alpha \delta+\beta \gamma-q^{2}\right) & +C \cdot 2 \gamma \delta=0, \\
A \beta^{2}+B \beta \delta & +C\left(\delta^{2}-q^{2}\right)=0 .
\end{array}
$$

From the first and second of these we have

$$
A: B: C=\gamma^{2}\left(\nabla+q^{2}\right):-2 \gamma \alpha \nabla+2 \gamma \delta q^{2}: \alpha^{2} \nabla-\left(\alpha^{2}+\alpha \delta+\beta \gamma\right) q^{2}+q^{4} ;
$$

or, writing herein for $\nabla, q^{4}$ the values $q^{2}, q^{2}(\alpha \delta-\beta \gamma)$, the three expressions divide by $2 \gamma q^{2}$, and we have

$$
A: B: C=\gamma: \delta-\alpha:-\beta .
$$

Combining these values in the first place with the equation $\alpha+\delta=-2 q$, we may write

$$
\alpha, \beta, \gamma, \delta=-q-p B,-2 p C, 2 p A,-q+p B,
$$

where $p$ is to be determined. Substituting in the last of the three equations, we have

that is,

$$
\text { A. } 4 p^{2} C^{2}-2 p B C(-q+p B)+C\left(-2 p q B+p^{2} B^{2}\right)=0,
$$

$$
p^{2} C\left(4 A C-B^{2}\right),=-p^{2} . C \Omega,=0,
$$

and the form $\left(a, b, c, d \gamma(x, y)^{3}\right.$ being arbitrary, neither $C$ nor $\Omega$ is $=0$; whence $p=0$, and the values are $\alpha, \beta, \gamma, \delta=-q, 0,0,-q$, that is,

a trivial result.

$$
(a, b, c, d \gamma-q x,-q y)^{3}=-q^{3} \cdot\left(a, b, c, d \gamma(x, y)^{3},\right.
$$


But, combining the same values with $\alpha+\delta=q$, we have

$$
\alpha, \beta, \gamma, \delta=\frac{1}{2} q-p B,-2 p C, 2 p A, \frac{1}{2} q+p B ;
$$

and then, substituting in the third equation, we have

that is,

$$
\text { A. } 4 p^{2} C^{2}-2 p B C\left(\frac{1}{2} q+p B\right)+C\left(-\frac{3}{4} q^{2}+p q B+p^{2} B^{2}\right)=0
$$

$$
C\left\{\left(4 A C-B^{2}\right) p^{2}-\frac{3}{4} q^{2}\right\}=0,
$$

or, omitting the factor $C$, and introducing the foregoing notation $\lambda^{2}=-\frac{1}{3}$, this is

$$
4 \Omega \lambda^{2} p^{2}-q^{2}=0 \text {, or say } p=\frac{1}{2 \lambda \sqrt{ } \Omega} q .
$$

For the unimodular substitution $\alpha \delta-\beta \gamma=1$, we must have $q^{2}=1$ : but, the transformation being

$$
(a, b, c, d \gamma \alpha x+\beta y, \gamma x+\delta y)^{3}=-q^{3} \cdot\left(a, b, c, d \gamma(x, y)^{3},\right.
$$

to make this strictly automorphic, we must have $q^{3}=-1$, and the two conditions are satisfied by $q=-1$; we then have $p=-\frac{1}{2 \lambda \sqrt{ } \Omega}$; and the coefficients are

$$
\alpha, \beta, \gamma, \delta,=\frac{-\lambda \sqrt{ } \Omega+B}{2 \lambda \sqrt{ } \Omega}, \frac{2 C}{2 \lambda \sqrt{ } \Omega}, \frac{-2 A}{2 \lambda \sqrt{ } \Omega}, \frac{-\lambda \sqrt{ } \Omega-B}{2 \lambda \sqrt{ } \Omega}
$$

which are the values given at the beginning of the paper, and which belong to the automorphic transformation

$$
(a, b, c, d \gamma \alpha x+\beta y, \gamma x+\delta y)^{3}=\left(a, b, c, d \gamma(x, y)^{3} .\right.
$$

The $\grave{a}$ priori reason for the periodicity-equation $\phi^{3} x=x$, is best seen by expressing the cubic function as a product of factors

$$
M(x-\mathrm{a} y)(x-\mathrm{b} y)(x-\mathrm{c} y) .
$$

The substitution must, it is clear, cyclically interchange these factors, and therefore, when performed three times in succession on any one of these factors, or consequently upon an arbitrary linear factor $x-\mathrm{f} y$, must leave the factor unaltered, and it must thus be a periodic substitution $\phi^{3} x=x$. But it was interesting to see how the condition for this, $\alpha^{2}+\delta^{2}+\alpha \delta+\beta \gamma=0$, comes out from the consideration of the equation

$$
(a, b, c, d \gamma \alpha x+\beta y, \gamma x+\delta y)^{3}=\left(a, b, c, d \gamma(x, y)^{3} .\right.
$$

